Revue d'histoire de l'Amérique française

REVUE D.HISTOIRE DE L'AMÉRIQUE FRANÇAISE

\title{
Le personnel politique de Montréal, 1880-1914 : évolution d'une élite municipale
}

\section{Paul-André Linteau}

Volume 52, numéro 2, automne 1998

URI : https://id.erudit.org/iderudit/005535ar

DOI : https://doi.org/10.7202/005535ar

Aller au sommaire du numéro

Éditeur(s)

Institut d'histoire de l'Amérique française

ISSN

0035-2357 (imprimé)

1492-1383 (numérique)

Découvrir la revue

Citer cet article

Linteau, P.-A. (1998). Le personnel politique de Montréal, 1880-1914 : évolution d'une élite municipale. Revue d'histoire de l'Amérique française, 52(2), 189-215. https://doi.org/10.7202/005535ar
Résumé de l'article

Entre 1880 et 1914, 234 hommes sont élus au Conseil municipal de Montréal, à titre de conseiller, maire ou commissaire. Grâce à la biographie collective, il est possible de faire ressortir certaines caractéristiques de cette élite politique. La supériorité numérique croissante des francophones se manifeste, tandis que le recul des anglophones affecte particulièrement les Irlandais. Malgré le poids croissant des membres des professions libérales, les hommes d'affaires dominent tout au long de la période, aussi bien parmi les francophones que chez les anglophones. L'étude permet en outre de dégager certains traits de la carrière politique municipale: longévité et accès à la présidence d'un comité pour un grand nombre de conseillers, mais aussi brièveté du passage au Conseil pour la moitié d'entre eux et présence d'au moins le quart des élus sur la scène provinciale ou fédérale.
Tous droits réservés @ Institut d'histoire de l'Amérique française, 1998
Ce document est protégé par la loi sur le droit d'auteur. L'utilisation des services d'Érudit (y compris la reproduction) est assujettie à sa politique d'utilisation que vous pouvez consulter en ligne.

https://apropos.erudit.org/fr/usagers/politique-dutilisation/ 


\title{
LE PERSONNEL POLITIQUE DE MONTRÉAL, 1880-1914: ÉVOLUTION D'UNE ÉLITE MUNICIPALE ${ }^{1}$
}

\author{
PAUL-ANDRÉ LINTEAU \\ Département d'histoire \\ Université du Québec à Montréal
}

\begin{abstract}
RÉSUMÉ
Entre 1880 et 1914, 234 hommes sont élus au Conseil municipal de Montréal, à titre de conseiller, maire ou commissaire. Grâce à la biographie collective, il est possible de faire ressortir certaines caractéristiques de cette élite politique. La supériorité numérique croissante des francophones se manifeste, tandis que le recul des anglophones affecte particulièrement les Irlandais. Malgré le poids croissant des membres des professions libérales, les hommes d'affaires dominent tout au long de la période, aussi bien parmi les francophones que chez les anglophones. L'étude permet en outre de dégager certains traits de la carrière politique municipale: longévité et accès à la présidence d'un comité pour un grand nombre de conseillers, mais aussi brièveté du passage au Conseil pour la moitié d'entre eux et présence d'au moins le quart des élus sur la scène provinciale ou fédérale.
\end{abstract}

\begin{abstract}
In Montreal, between 1880 and 1914, 234 men were elected to the City Council as alderman, mayor or commissioner. A prosopographic study highlights some characters of this political elite. Francophones' rising numerical domination is obvious while the anglophones' decline is more pronounced among the Irish. Throughout the period businessmen are in the majority, among both francophones and anglophones, despite the professionals' growing share. Some features of the municipal political career are also identified. Many aldermen enjoy a political longevity coupled with the presidency of a committee, but half of them experience rather short terms of office. At least a quarter of the group is involved actively in provincial or federal politics.
\end{abstract}

Entre 1880 et 1914, la scène politique municipale à Montréal connaît des années mouvementées, caractérisées par le long combat qui oppose populistes et réformistes et par l'emprise croissante des francophones. Le

1. Version révisée d'une communication présentée au congrès de la Société historique du Canada, en août 1995. La recherche à la base de ce texte a pu être réalisée grâce à une subvention du Conseil de recherches en sciences humaines du Canada. Je remercie Jocelyne Savaria et Christine Simard qui ont fait la collecte et la saisie des données et François Guérard qui a mené les traitements informatiques. 
Conseil municipal représente alors un lieu de pouvoir vivement recherché et contesté. En outre, les dimensions de ce champ de bataille sont considérablement modifiées: l'annexion successive d'une vingtaine de municipalités élargit ses assises territoriales, tandis qu'une démocratisation accrue étend ses assises électorales ${ }^{2}$.

Une telle animation se déroule sur un fond de forte croissance. La population fait plus que tripler entre 1881 et 1911, celle de la ville passant de 140747 à 467 986, celle de l'agglomération de 170745 à 528397. S'appuyant sur le port et les chemins de fer, sur une base industrielle diversifiée et sur de nombreuses institutions financières, l'économie de la ville est en expansion. Montréal est alors au faîte de sa puissance comme métropole du Canada.

Les transformations qui en résultent ne vont pas sans tensions. Une imposante masse ouvrière vit péniblement les effets de l'industrialisation et de la prolétarisation. L'émergence du syndicalisme lui donne une voix, certes minoritaire, qui s'exprime tout de même sur la scène politique au début du $\mathrm{XX}^{\mathrm{e}}$ siècle, en même temps que l'ouverture du système politique lui permet d'avoir un poids électoral accru. Par ailleurs, les tensions ethniques, qui atteignent leur paroxysme en 1885 avec l'affaire Riel et l'épidémie de variole, restent une composante importante de la vie publique montréalaise, bien que les relations entre les communautés comprennent également une bonne dose de compromis, d'échanges et de collaboration ${ }^{3}$.

Parmi les éléments de ce contexte général, il faut en outre souligner l'essor des élites montréalaises. Ce phénomène, au cœur de la présente étude, présente au moins trois facettes notables. Il y a d'abord l'expansion considérable des effectifs des milieux d'affaires, favorisée par la croissance économique et démographique. Il faut ici se dégager de l'image du resserrement du recrutement de l'élite économique qu'a livrée Acheson et qui ne concerne que la couche supérieure de la bourgeoisie du secteur manufacturier ${ }^{4}$. Des milliers de nouveaux entrepreneurs - associés à la PME -, de promoteurs et de commerçants locaux émergent en effet à la faveur de l'expansion qui caractérise la période. Montréal leur offre un grand potentiel d'occasions à saisir.

2. Paul-André Linteau, Histoire de Montréal depuis la Confédération (Montréal, Boréal, 1992), 119-130, 253-268; Michèle Dagenais, La démocratie à Montréal de 1850 à nos jours (Montréal, Ville de Montréal, 1992), 21-25.

3. Sur l'évolution de Montréal pendant cette période, voir P.-A. Linteau, Histoire de Montréal..., 11-277; voir aussi Robert Rumilly, Histoire de Montréal (Montréal, Fides, 1972), 3: 524 p.

4. T. W. Acheson, «Changing Social Origins of the Canadian Industrial Elite, 1880-1910», Glenn Porter et Robert Cuff, dir., Enterprise and National Development: Essays in Canadian Business and Economic History (Toronto, Hakkert, 1973), 51-79. 
On relève également l'importance croissante du groupe des spécialistes et des experts. Les membres des professions libérales, notamment les avocats, les médecins et les ingénieurs, ainsi que les cadres supérieurs des grandes entreprises acquièrent une visibilité accrue dans la société montréalaise et deviennent des acteurs de poids dans les débats économiques et sociaux. Le mouvement hygiéniste en fournit un bon exemple dans le cas des médecins.

De façon plus spécifique, il faut noter enfin l'émergence de nouvelles élites francophones. Particulièrement remarquable est l'essor d'une bourgeoisie d'affaires qui développe un grand nombre d'entreprises francophones et dont les efforts sont appuyés par une Chambre de commerce (1887) et par une presse d'affaires. Ces nouvelles élites francophones sont aussi visibles dans les professions libérales et dans le monde des médias ${ }^{5}$.

Dans quelle mesure la scène politique locale reflète-t-elle ces phénomènes sociaux et en particulier l'émergence de forces nouvelles à Montréal? Quelle place y occupent les milieux d'affaires en expansion? Quel rôle y joue le nouveau groupe des spécialistes et des experts? Comment la classe ouvrière y fait-elle entendre sa voix? Comment la poussée démographique des francophones dans la ville se manifeste-t-elle au conseil municipal? Leur présence est-elle différente de celle des anglophones? Et ces derniers, qui sont-ils? D'où viennent les uns et les autres?

La présente étude vise à apporter des éléments de réponses à ces questions. S'appuyant sur une biographie collective des élus municipaux, elle examine quelques aspects de la composition ethnolinguistique et sociale de l'élite politique municipale et de son évolution au cours de la période. Elle se penche aussi sur certaines caractéristiques de la carrière politique sur la scène municipale. Diffère-t-elle de celle qui prévaut aux niveaux provincial et fédéral? Offre-t-elle un simple tremplin vers d'autres niveaux de gouvernements ou attire-t-elle des individus qui sont prêts à lui consacrer une partie importante de leur vie? Avant de préciser les paramètres de cette étude

5. Paul-André Linteau, René Durocher et Jean-Claude Robert, Histoire du Québec contemporain, 1: De la Confédération à la crise (1867-1929) (Montréal, Boréal, 1979 et 1989), chap. 8 et 25; Paul-André Linteau, «Rapports de pouvoir et émergence d'une nouvelle élite canadienne-française à Montréal, 1880-1914», Études canadiennes/Canadian Studies. Revue interdisciplinaire des études canadiennes en France, 21,1 (1986): 163-172; Yves Bélanger et Pierre Fournier, L'entreprise québécoise. Développement historique et dynamique contemporaine (Montréal, Hurtubise HMH, 1987), 2045; Ronald Rudin, Banking en français. Les banques canadiennes-françaises, 1835-1925 (Montréal, Boréal, 1988), 244 p.; Fernande Roy, Progrès, harmonie, liberté. Le libéralisme des milieux d'affaires francophones à Montréal au tournant du siècle (Montréal, Boréal, 1988), 301 p.; Robert C. H. Sweeny, «Un effort collecctif québécois: la création, au début du $\mathrm{XX}^{\mathrm{e}}$ siècle, d'un marché privé et institutionnalisé de capitaux», Revue d'histoire de l'Amérique française, 49,1 (été 1995): 35-72. 
et de livrer de premiers résultats, il faut toutefois faire le point sur l'état des connaissances en ce domaine.

\section{L'ÉTUDE DE LA SCÈNE POLITIQUE MONTRÉALAISE}

Depuis un quart de siècle, l'étude de la scène politique montréalaise de la période 1880-1914 a été dominée par le thème de l'opposition entre réformistes et populistes. Les travaux de D. J. Russell, Michel Gauvin et

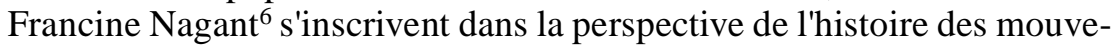
ments réformistes américains. Ils mettent l'accent sur les orientations, les politiques et les stratégies des acteurs. Sauf chez Russell, qui fait une biographie de $\mathrm{H}$. B. Ames, ils ne mènent pas une analyse systématique de la carrière de ces acteurs. Il en est de même dans le cas d'Annick Germain ${ }^{7}$ qui, de son côté, soutient la thèse de l'émergence d'une scène politique municipale autonome par rapport à la sphère économique, scène dont elle souligne avec justesse la complexité. Elle a toutefois tendance à sousestimer les assises économiques de l'élite politique francophone. Sur un autre plan, les enjeux administratifs de la politique montréalaise ont été abordés par Michèle Dagenais ${ }^{8}$.

L'action politique ouvrière qui se manifeste à Montréal au début du $\mathrm{XX}^{\mathrm{e}}$ siècle a fait l'objet des travaux d'une équipe d'historiens de l'Université du Québec à Montréal, ainsi que de Jacques Rouillard ${ }^{9}$, outre ceux déjà cités d'Annick Germain. Si la présence ouvrière sur la scène munici-

6. D. J. Russell, H. B. Ames as Municipal Reformer, mémoire de maîtrise (histoire), Université McGill, 1971; Michel Gauvin, The Municipal Reform Movement in Montreal, 1886-1914, mémoire de maîtrise (histoire), Université d'Ottawa, 1972, 163 p.; Id., «The Reformer and the Machine: Montreal Civic Politics From Raymond Préfontaine to Médéric Martin», Journal of Canadian Studies/Revue d'études canadiennes, 13,2 (été 1978): 16-26; Francine Nagant, La politique municipale à Montréal 1910-1914: l'échec des réformistes et le triomphe de Médéric Martin, mémoire de maîtrise (histoire), Université de Montréal, 1982, 297 p.

7. Annick Germain, Les mouvements de réforme urbaine à Montréal au tournant du siècle. Modes de développement, modes d'urbanisation et transformation de la scène politique (Montréal, Centre d'information et d'aide à la recherche, Département de sociologie, Université de Montréal, 1984), 415 p.; Id., «L'émergence d'une scène politique: mouvement ouvrier et mouvements de réforme urbaine à Montréal au tournant du siècle — Essai d'interprétation», Revue d'histoire de l'Amérique française, 37,2 (septembre 1983): 185-199.

8. Michèle Dagenais, Dynamiques d'une bureaucratie. L'administration municipale de Montréal et ses fonctionnaires, 1900-1945, thèse de doctorat (histoire), Université du Québec à Montréal, 1992, 413 p.

9. Groupe de recherches sur l'histoire des mouvements politiques ouvriers de l'Université du Québec à Montréal, Chronologie des mouvements politiques ouvriers au Québec de la fin du $19^{e}$ siècle jusqu'à 1919 (RCHTQ, 1976), 293 p.; Id., L'action politique des ouvriers québécois (fin du XIX siècle à 1919). Recueil de documents (Montréal, Presses de l'Université du Québec, 1976), 176 p. Jacques Rouillard, «L'action politique ouvrière au début du $20^{\mathrm{e}}$ siècle», dans Fernand Harvey, dir., Le mouvement ouvrier au Québec (Montréal, Boréal, 1980), 185-213. 
pale y est brièvement examinée, ni les élus ni leurs interventions au Conseil ne sont étudiés.

La seule étude qui s'apparente à la nôtre est l'analyse prosopographique qu'a produite il y a plus de trente ans par le politologue Guy Bourassa ${ }^{10}$. Ce dernier distingue trois périodes dans l'évolution du personnel politique montréalais. Pendant la première, de 1840 à 1873, Montréal serait dirigée par une aristocratie financière, majoritairement d'origine britannique. La seconde, de 1873 à 1914, en serait une de transition, marquée par l'affirmation des francophones et par l'émergence de membres des professions libérales et d'«administrateurs moyens», ce qui donnerait à l'élite politique un caractère plus hétérogène. La richesse ne serait plus la seule ressource politique, alors que «le rayonnement intellectuel, le nombre et la puissance ethnique deviennent des atouts de première force ${ }^{11}{ }$. La troisième période, de 1914 à 1960, serait caractérisée par la professionnalisation du personnel politique, désormais plus près de ses électeurs. La deuxième période identifiée par Bourassa correspond d'assez près à la nôtre, ce qui rend la comparaison particulièrement intéressante. Il faut dire toutefois que l'étude de Bourassa reste plutôt globale ${ }^{12}$ et que les travaux réalisés depuis sur l'histoire de Montréal permettent d'examiner cette question d'un autre œil.

Un ouvrage récent sur les maires de Montréal n'apporte guère d'eau au moulin. Les notices, d'abord rédigées pour un quotidien, font peu de place à la biographie des personnages et se concentrent sur les événements survenus pendant leur mandat à la mairie ${ }^{13}$.

La recherche prosopographique que nous avons entreprise devrait donc permettre d'ajouter à la connaissance de la scène politique municipale. Un dossier biographique a été constitué pour chacun des 234 hommes qui ont occupé un poste de maire, de conseiller municipal ou de commissaire à l'Hôtel de Ville de Montréal entre les élections de 1880 et celles de 1914 (en excluant ces dernières). Parmi eux, on compte 16 maires (dont 11 ont aussi été conseillers), 224 conseillers - souvent appelés échevins - et 5 commissaires $^{14}$.

10. Guy Bourassa, «Les élites politiques de Montréal: de l'aristocratie à la démocratie», Canadian Journal of Economics and Political Science/Revue canadienne d'économique et de science politique, 31,1 (février 1965): 35-51, reproduit dans Richard Desrosiers, dir., Le personnel politique québécois (Montréal, Boréal, 1972), 117-142; nous utilisons cette dernière version.

11. Ibid., 126.

12. Elle ne contient d'ailleurs aucun tableau de données et reste imprécise sur le plan de la méthodologie.

13. Claude-V. Marsolais, Luc Desrochers et Robert Comeau, Histoire des maires de Montréal (Montréal, VLB éditeur, 1993), 323 p.

14. Le Bureau des commissaires est formé en 1910. 
Les dossiers individuels ont été établis à partir d'un éventail varié de sources. Les répertoires biographiques, dont on trouvera une liste en annexe, ont fourni la matière première; il faut en particulier souligner l'utilité de l'ouvrage de Cléophas Lamothe qui contient une notice biographique pour tous les maires et pour une partie des conseillers élus entre 1840 et $1903^{15}$. Ont été ensuite utilisés, aux Archives municipales de Montréal, les dossiers biographiques ainsi que les listes des membres du Conseil et de ses comités. Les annuaires Lovell et les listes nominatives des recensements de 1871, 1881 et 1901 ont été dépouillés systématiquement. Des recherches ponctuelles ont été faites dans les rôles d'évaluation et des valeurs locatives et dans les registres d'état civil. Les informations contenues dans les dossiers biographiques ont ensuite été versées dans un fichier informatique standardisé.

Les premiers résultats de cette recherche, présentés ici, sont axés sur trois sujets: les origines ethniques, linguistiques et régionales; les caractéristiques professionnelles; les principaux paramètres de la carrière politique. Ce sont là des variables pour lesquelles les informations sont assez complètes. Les données ont été analysées selon trois regroupements: d'abord pour l'ensemble du personnel politique, quel que soit le poste occupé; puis pour les seuls conseillers, en distinguant deux sous-groupes: ceux qui ont été élus pour la première fois avant 1898 et ceux qui l'ont été en 1898 ou après ${ }^{16}$; enfin, pour tous les membres du Conseil (maire et conseillers) à certaines années choisies $(1881,1888,1894,1900,1908$ et $1912)^{17}$.

\section{LES ORIGINES}

L'origine ethnique et linguistique est une dimension fondamentale de la politique municipale à Montréal. Il est bien connu que les francophones, majoritaires dans la ville depuis le milieu des années 1860, doivent attendre jusqu'en 1882 pour obtenir une majorité d'un siège au Conseil et que leur emprise s'accroît de façon notable par la suite. L'enquête a permis de pousser plus loin l'analyse de ce phénomène. Pour l'ensemble de la période étudiée (tableau 1), la part des Canadiens français parmi le personnel politique atteint $62,4 \%$, ce qui correspond assez bien à leur poids

15. J. Cléophas Lamothe, Histoire de la Corporation de la Cité de Montréal depuis ses origines jusqu'à nos jours (Montréal, Montreal Printing and Publishing Co., 1903), 848 p.

16. L'année 1898 a été retenue parce qu'elle se situe à mi-chemin de la période étudiée et qu'elle marque le début du renversement de l'organisation politique de Raymond Préfontaine par les réformistes et donc d'un renouvellement du personnel politique.

17. Ces trois regroupements, utilisés dans le texte, ne sont pas tous présents dans les tableaux; le nombre de ceux-ci a été volontairement restreint pour ne pas alourdir la démonstration. 
démographique dans la ville, qui passe de $56,1 \%$ en 1881 à $63,5 \%$ en 1911. Celle du personnel d'origine britannique atteint $36,7 \%$ : on compte 36 Irlandais, 31 Anglais, 18 Écossais et un autre Britannique dont l'origine précise n'a pu être déterminée. La présence irlandaise au Conseil municipal est tangible $(15,4 \%)$ et elle représente un phénomène qui, à notre connaissance, n'a jamais été mis en lumière. Rappelons que la proportion des Montréalais d'origine britannique est de 41,4\% en 1881 et de $25,7 \%$ en $1911^{18}$. Notons aussi l'élection, en fin de période, d'un représentant de chacun des deux plus importants autres groupes ethniques: un Juif russe et un Italien.

TABLEAU 1

\section{ORIGINE ETHNIQUE ET GROUPE LINGUISTIQUE PERSONNEL POLITIQUE MUNICIPAL DE MONTRÉAL 1880-1914}

\begin{tabular}{|c|c|c|c|c|}
\hline Origine ethnique & $\begin{array}{c}\text { Groupe } \\
\text { anglophone }\end{array}$ & $\begin{array}{c}\text { Groupe } \\
\text { francophone }\end{array}$ & Total & $\%$ du tota \\
\hline Anglaise & 27 & 4 & 31 & 13,2 \\
\hline $\begin{array}{l}\text { Britannique } \\
\text { indéterminée }\end{array}$ & 1 & & 1 & 0,4 \\
\hline Écossaise & 18 & & 18 & 7,7 \\
\hline Française & & 146 & 146 & 62,4 \\
\hline Irlandaise & 35 & 1 & 36 & 15,4 \\
\hline Italienne & 1 & & 1 & 0,4 \\
\hline Russe & 1 & & 1 & 0,4 \\
\hline TOTAL & 83 & 151 & 234 & 100,0 \\
\hline$\%$ du total & 35,5 & 64,5 & 100 & \\
\hline
\end{tabular}

Cette répartition évolue de façon notable au cours de la période, comme le montre l'examen des années choisies (tableau 2). En 1881, le Conseil de 28 membres n'en compte que 12 d'origine française, une nette sous-représentation ${ }^{19}$. Les Irlandais, qui forment alors la moitié de la population britannique de la ville, ont 8 représentants, les Anglais et les Écossais, 4 chacun. En 1888, les Canadiens français ont plus de la moitié des sièges et leur poids s'accroît surtout au détriment des Anglais et des Écos-

18. Sur la répartition des groupes ethniques à Montréal, voir Paul-André Linteau, «La montée du cosmopolitisme montréalais», Questions de culture, 2 (1982): 23-53.

19. Notons toutefois que deux des individus d'origine britannique sont considérés comme des francophones, ce qui donne à ces derniers une représentation de $50 \%$. 
sais, tandis que les Irlandais maintiennent leur position. À partir de la décennie suivante, les Canadiens français sont légèrement surreprésentés et monopolisent les deux tiers des postes en 1908 et 1912. L'ampleur de ce renversement apparaît encore plus quand on prend en compte l'année d'accession au Conseil: seulement 55\% des conseillers élus pour la première fois avant 1898 sont d'origine française, tandis qu'ils sont près de $72 \%$ à partir de cette date. Par ailleurs, la présence irlandaise décline de façon nette dans la dernière décennie du siècle (tableau 2). Les données sur la première élection le confirment: les Irlandais comptent pour $21 \%$ des nouveaux élus avant 1898 et pour seulement $8,5 \%$ à partir de ce moment. Cela n'étonne guère, puisque la part de leurs effectifs dans la population de la ville est en chute rapide. Le poids des Anglais et Écossais est plus fluctuant d'une année à l'autre, mais il est lui aussi en déclin, quoique de façon moins nette que chez les Irlandais. Ensemble, ces deux groupes passent de près de $24 \%$ des nouveaux élus avant 1898 à $18 \%$ par la suite.

TABLEAU 2

\section{ORIGINE ETHNIQUE \\ MEMBRES DU CONSEIL MUNICIPAL DE MONTRÉAL ANNÉES CHOISIES, 1881-1912}

$\begin{array}{lrrrrrr} & 1881 & 1888 & 1894 & 1900 & 1908 & 1912 \\ \text { Anglaise } & 4 & 4 & 1 & 5 & 6 & 2 \\ \begin{array}{l}\text { Britannique } \\ \text { indéterminée }\end{array} & & & 1 & 1 & 1 & \\ \text { Écossaise } & 4 & 2 & 2 & 2 & 3 & 5 \\ \text { Française } & 12 & 21 & 17 & 22 & 28 & 22 \\ \text { Irlandaise } & 8 & 10 & 5 & 4 & 4 & 2 \\ \text { Italienne } & & & & & & 1 \\ \text { Russe } & 28 & 37 & 26 & 34 & 42 & 33 \\ \text { TOTAL } & & & & & & 1\end{array}$

Plusieurs facteurs peuvent expliquer ces fluctuations. La montée des Canadiens français est alimentée par la croissance de leurs effectifs dans la ville, par les nombreuses annexions de municipalités de banlieue et par l'ouverture du système électoral ${ }^{20}$. Pour les autres groupes, la concentra-

20. Rappelons par exemple que, jusqu'en 1887, le non-paiement de la taxe de la corvée faisait perdre leur droit de vote à de nombreux petits électeurs, surtout canadiens-français. Cette contrainte est supprimée par la loi 50 Vict. (1887) chap. 56. 
tion en certains points du territoire est à prendre en compte. Il faut aussi se rappeler que les circonscriptions électorales sont de taille très inégale. Pour pousser plus loin l'explication, il faudrait faire une analyse détaillée de la représentation, quartier par quartier, et examiner l'effet des nouveaux découpages territoriaux qui surviennent en cours de route.

La composition ethnique évolue-t-elle de la même façon pour les maires? Le prestige associé à cette fonction conduit-il à une lutte serrée entre les deux blocs ethniques? Depuis longtemps, une règle non écrite veut qu'il y ait alternance à ce poste, mais elle n'est pas toujours rigoureusement respectée. Ainsi, pendant la période, dix des maires sont d'origine française, tandis que seulement six sont d'origine britannique. Cela donne une répartition $(62,5 \%$ et $37,5 \%)$ très proche de celle qui a été calculée pour l'ensemble du personnel politique. La divergence se manifeste plutôt dans la temporalité du phénomène. Ainsi, de 1880 à 1887, alors que l'élément britannique est encore nombreux au Conseil, les maires sont tous d'origine française (cette séquence de domination francophone a même débuté en 1877). Par la suite, on retourne à une certaine alternance qui devient absolument parfaite entre 1902 et 1914, à une époque où la domination du Conseil par les Canadiens français est fermement établie. On peut y voir un indice du caractère plus honorifique de la fonction de maire: le pouvoir véritable est entre les mains des conseillers et on peut se permettre de donner une image d'ouverture ou d'harmonie ethnique en la personne du premier magistrat. Quoi qu'il en soit, ce régime prendra fin en 1914. L'élection de Médéric Martin à la mairie vient alors briser définitivement l'alternance et, pendant le reste $\mathrm{du} \mathrm{XX}^{\mathrm{e}}$ siècle, tous les maires seront d'origine française. Cela explique que notre étude s'arrête à la veille des élections de 1914, habituellement perçues comme un important point de rupture dans l'histoire politique de Montréal.

Les limites imposées par la définition de l'origine ethnique dans les recensements canadiens (l'origine du premier ancêtre de sexe masculin arrivé en Amérique) nous ont amené à examiner la question de l'appartenance linguistique du personnel politique (tableau 1). Divers indices ${ }^{21} \mathrm{de}$ transfert linguistique nous ont permis de constater que cinq hommes politiques d'origine britannique (quatre Anglais et un Irlandais) appartiennent en fait ou sont perçus comme appartenant au groupe francophone. Par ailleurs, les deux individus d'autres origines sont identifiés au groupe anglophone. Cette répartition permet de constater que, pour l'ensemble du personnel politique, $64,5 \%$ des effectifs sont francophones et $35,5 \%$ anglo-

21. Parmi ces indices notons l'origine de la mère, la réponse «origine française» parfois donnée au recenseur, les études en français, la carrière en milieu francophone et la langue de la notice biographique dans l'ouvrage de Lamothe, ce dernier rédigeant chacune d'elles dans la langue du sujet. 
phones. La part des francophones passe de $57,6 \%$ chez les nouveaux conseillers élus avant 1898 à 73,6\% chez ceux qui le sont depuis cette date; celle des anglophones, de 42,4\% à 26,4\%. Dans la suite de notre analyse, nous nous reférerons surtout au groupe linguistique, tout en examinant à l'occasion les spécificités de l'origine ethnique.

Sur le plan de l'appartenance religieuse, comme on peut s'y attendre, les catholiques forment la majorité (tableau 3) et leur proportion augmente avec la francisation du Conseil (68\% des nouveaux élus avant 1898 et $83 \%$ depuis). Parmi eux, 6 sont d'origine anglaise et 3 d'origine écossaise. Seulement 21 des 36 Irlandais sont catholiques, tandis que les 15 autres appartiennent aux différents groupes protestants.

TABLEAU 3

\section{RELIGION}

PERSONNEL POLITIQUE MUNICIPAL DE MONTRÉAL 1880-1914

$\begin{array}{lrr}\text { Religion } & \text { Nombre } & \% \\ \text { Anglicane } & 17 & 7,2 \\ \text { Baptiste } & 2 & 0,9 \\ \text { Catholique } & 174 & 74,4 \\ \text { Congrégationaliste } & 1 & 0,4 \\ \text { Épiscopalienne } & 2 & 0,9 \\ \text { Juive } & 1 & 0,4 \\ \text { Méthodiste } & 9 & 3,8 \\ \text { Presbytérienne } & 22 & 9,4 \\ \text { Protestante } & 1 & 0,4 \\ \text { Unitarienne } & 2 & 0,9 \\ \text { Non identifiée } & 3 & 1,3 \\ \text { TOTAL } & 234 & 100,0\end{array}$

L'examen du pays de naissance (ou de la province dans le cas du Canada) permet de constater que les immigrants forment moins du cinquième des effectifs (tableau 4). Les îles Britanniques fournissent le plus gros de ce contingent, réparti pour moitié entre l'Irlande, d'une part, et l'Angleterre et l'Écosse, d'autre part; viennent ensuite les États-Unis. Les individus nés dans les provinces ou colonies d'Amérique du Nord britannique autres que le Québec sont peu nombreux. Le Québec domine nettement comme lieu d'origine, avec $81,2 \%$ du total. Sa part, qui n'est que de $61 \%$ en 1881 , se situe autour de $90 \%$ au début du $\mathrm{XX}^{\mathrm{e}}$ siècle. L'immigration repré- 
sente donc un facteur de moins en moins important dans la formation de l'élite politique municipale. Cela reflète sans doute le fait que, dans les dernières décennies du $\mathrm{XIX}^{\mathrm{e}}$ siècle, l'immigration contribue beaucoup moins qu'antérieurement à la croissance de la population montréalaise, tandis que les migrations internes ont un poids plus considérable.

TABLEAU 4

\section{PAYS OU PROVINCE DE NAISSANCE PERSONNEL POLITIQUE MUNICIPAL DE MONTRÉAL 1880-1914}

$\begin{array}{lrcrc}\text { Pays } & \text { Anglophones } & \text { Francophones } & \text { Total } & \text { \% du total } \\ \text { Angleterre } & 6 & & 6 & 2,6 \\ \text { Argentine } & 1 & & 1 & 0,4 \\ \text { Écosse } & 8 & & 8 & 3,4 \\ \text { États-Unis } & 8 & 1 & 9 & 3,8 \\ \text { Irlande } & 14 & & 14 & 6,0 \\ \text { Nouvelle-Écosse } & 1 & & 1 & 0,4 \\ \text { Ontario } & 4 & & 4 & 1,7 \\ \text { Québec } & 40 & 150 & 190 & 81,2 \\ \text { Terre-Neuve } & 1 & & 1 & 0,4 \\ \text { TOTAL } & 83 & 151 & 234 & 100,0\end{array}$

Seule une minorité, cependant, est née à Montréal (54) ou dans le reste de l'île (16), soit 30\% du total du personnel politique et 37\% des Québécois de naissance. Le plus grand nombre (84) provient de la plaine de Montréal, hors de lîle, soit 36\% du total et $44 \%$ des Québécois. C'est là une indication du rôle extrêmement important que joue alors cette région dans la migration rurale vers Montréal. Un peu plus de la moitié de ce dernier groupe est originaire de la rive nord. Le reste du Québec ne fournit que 23 individus, tandis que la localité de naissance de 13 autres Québécois est inconnue.

L'analyse des origines ethnolinguistiques confirme, tout en précisant ses rythmes et ses contours, la montée des francophones au Conseil municipal. Elle permet d'identifier un point de rupture important vers la fin des années 1880: par la suite, la part des Britanniques, notamment celle des Irlandais, décline ou piétine ${ }^{22}$. Elle attire l'attention sur les différences qui existent entre le groupe des maires et l'ensemble du personnel politique.

22. Guy Bourassa, 130, prétend que la montée des Canadiens français «se fait surtout au détriment du groupe écossais», mais notre analyse montre que la situation est plus complexe et que ce sont les Irlandais qui reculent le plus. 
L'analyse met aussi en lumière l'origine essentiellement québécoise des membres du Conseil. Elle précise le phénomène de la migration rurale en montrant qu'il est avant tout régional, la métropole et son hinterland immédiat fournissant les deux tiers des élus et les quatre cinquièmes de ceux qui sont originaires de la province.

\section{LES CARACTÉRISTIQUES PROFESSIONNELLES}

L'appartenance sociale représente une autre caractéristique fondamentale pour l'étude du personnel politique. Rappelons d'abord que le recrutement des élus est balisé par une contrainte importante. En effet, pendant presque toute la période, maires et conseillers sont soumis à l'exigence de la qualification foncière. Pour être candidats, les premiers doivent posséder des biens fonciers d'une valeur d'au moins $4000 \$$, somme qui est portée à $10000 \$$ à compter de 1892. Quant aux conseillers, ils doivent faire état de propriétés valant au moins $2000 \$$. C'est dire que dans une ville où seulement environ $15 \%$ des chefs de ménage sont propriétaires, cette exigence a un effet de sélection sociale marqué et limite le recrutement à une minorité de possédants. Les chefs syndicaux dénoncent d'ailleurs cette situation et réclament l'élimination de la qualification foncière. Inexistante pour les commissaires élus à partir de 1910, cette obligation est finalement abolie pour le maire et les conseillers en 1912.

Les études de prosopographie accordent souvent une grande importance à la profession du père afin de déterminer l'origine sociale des individus. Nos données ne sont pas suffisamment complètes pour pousser très loin une telle analyse. Cette information n'est connue que pour 123 des élus, soit à peine plus de la moitié. Parmi ceux-ci, on relève un peu plus du tiers de fils de cultivateurs et un sixième de fils d'artisans; $29 \%$ des pères sont des hommes d'affaires et $10 \%$ des membres des professions libérales. Il y aurait donc un peu plus d'élus d'origine modeste, mais aussi un nombre assez substantiel qui vient d'un milieu identifié à l'élite. Même partiels, ces résultats révèlent une mobilité sociale ascendante significative parmi l'élite politique montréalaise; il ne faut pas s'en surprendre car, pendant cette période de fort exode rural, la ville offre aux nouveaux venus des possibilités très différentes de celles de leur milieu d'origine. Il faudra compléter les informations et, surtout, comparer une à une les professions des pères et des fils pour arriver à mieux cerner le phénomène de mobilité sociale. Tel n'est pas l'objectif ici: nous nous intéressons plutôt à la position sociale des élus au moment où ils sont membres du Conseil municipal.

Le principal indice de cette position sociale est l'appartenance professionnelle. Beaucoup des individus qui forment les élites urbaines de la fin 
du XIX ${ }^{\mathrm{e}}$ siècle ont de multiples intérêts, dans des champs d'activité distincts, et occupent plus d'une profession au cours de leur carrière. Cette diversité a été prise en compte dans la constitution des fichiers biographiques et toutes les mentions professionnelles ont été relevées. L'analyse qui suit repose toutefois sur la profession principale de chaque membre du groupe, telle qu'elle apparaissait dans les sources.

Les professions individuelles ont été rassemblées par groupe professionnel (tableau 5). Une première constatation s'impose: la prépondérance des hommes d'affaires parmi le personnel politique montréalais. Les commerçants, les industriels, les entrepreneurs en construction et les autres hommes d'affaires représentent $72,6 \%$ des 234 individus. Cette prépondérance est cependant plus marquée chez les conseillers élus pour la première fois avant 1898 (82\%), que chez ceux élus en 1898 ou après (65\%). Le Conseil de 1881 comptait 22 hommes d'affaires parmi ses 28 membres, celui de 1912 en a 21 sur 33.

Tout au long de la période, les commerçants forment le groupe le plus nombreux, avec plus du tiers des effectifs. Parmi ces 85 individus, 37 vendent des produits alimentaires, principalement de l'épicerie, mais aussi des viandes, des vins et spiritueux ou des fruits, 11 travaillent dans le bois, le charbon ou les combustibles, 10 ont un commerce de nouveautés et 5 vendent des vêtements. À l'évidence, il y a parmi eux bon nombre de petits détaillants de quartier, mais au moins 21 sont clairement identifiés comme grossistes.

Viennent ensuite les industriels $(14,1 \%)$. Leur poids relatif tend à décliner puisque les deux tiers d'entre eux sont élus pour la première fois avant 1898. Ils sont même 11 sur 37 dans le Conseil de 1888, mais seulement 3 sur 33 dans celui de 1912. Ils sont à l'œuvre dans des secteurs très diversifiés, mais on en retrouve 5 dans la chaussure et 3 dans l'édition et l'imprimerie.

Les entrepreneurs en construction sont aussi nombreux que les industriels et leur présence se fait sentir tout au long de la période. Ils ont évidemment un intérêt particulier dans le développement urbain, ce qui peut expliquer leur participation politique. Il en est de même pour les sept courtiers ou promoteurs immobiliers qui représentent plus du tiers du groupe des autres hommes d'affaires. Il s'agit là seulement de ceux dont c'est la profession principale, mais il faut souligner qu'un grand nombre d'élus, tant chez les hommes d'affaires que chez les membres des professions libérales, sont aussi actifs dans le secteur immobilier. 
TABLEAU 5

\section{PROFESSION PRINCIPALE PAR GROUPE PROFESSIONNEL PERSONNEL POLITIQUE MUNICIPAL DE MONTRÉAL 1880-1914}

$\begin{array}{lrcrc}\text { Groupe } & \text { Anglophones } & \text { Francophones } & \text { Total } & \% \text { du total } \\ \text { Commerçants } & 31 & 54 & 85 & 36,3 \\ \text { Industriels } & 19 & 14 & 33 & 14,1 \\ \begin{array}{l}\text { Entrepreneur en } \\ \text { construction }\end{array} & 7 & 26 & 33 & 14,1 \\ \begin{array}{l}\text { Autres hommes } \\ \text { d'affaires }\end{array} & 7 & 12 & 19 & 8,1 \\ \text { Gestionnaires } & 3 & & & \\ \text { Membres des professions } & 13 & 43 & 56 & 23,9 \\ \text { libérales } & 2 & 1 & 3 & 1,3 \\ \text { Employés } & 1 & 1 & 2 & 0,9 \\ \text { Ouvriers et artisans } & 83 & 151 & 234 & 100,0 \\ \text { TOTAL } & & & & \end{array}$

Seulement deux des autres hommes d'affaires ont comme profession principale celle de financier ou banquier, mais là encore les sources révèlent que de nombreux membres du Conseil sont également présents dans ce secteur. Ainsi, au moins 19 d'entre eux sont membres d'un conseil d'administration d'une banque, tandis que plusieurs sont administrateurs de compagnies d'assurance ou de sociétés de fiducie.

La seconde grande catégorie représentée au Conseil est celle des membres des professions libérales, avec près du quart des effectifs totaux. Contrairement aux hommes d'affaires, leur poids augmente au $\mathrm{XX}^{\mathrm{e}}$ siècle. Ils ne représentent que $15 \%$ des conseillers élus pour la première fois avant 1898 , mais $32 \%$ de ceux qui le sont à partir de cette date. Le Conseil de 1881 en compte 5 , celui de 1912, 11. Les professions juridiques dominent sans surprise, avec 28 avocats et 6 notaires. Celles de la santé font aussi bonne figure: 14 médecins, 4 pharmaciens et un dentiste. Avec seulement deux ingénieurs, un architecte et aucun comptable, les autres professions libérales n'ont qu'une faible représentation.

Il faut souligner qu'il n'y a pas de division étanche entre le monde des affaires et celui des professions libérales. Le phénomène de l'avocat-brasseur d'affaires, par exemple, est encore très présent et touche une bonne douzaine des avocats. Parmi les plus célèbres, notons Raymond Préfontaine, que son mariage associe à la famille Rolland, Henri-Benjamin 
Rainville, très actif dans les entreprises d'électricité, Sévère Rivard et Joseph-Ulric Émard, deux promoteurs immobiliers, le premier à SaintJean Baptiste, le second à Ville-Émard. Au moins trois des six notaires sont également liés à la promotion immobilière. Les médecins paraissent plus centrés sur leur profession, mais au moins quatre d'entre eux sont aussi actifs en affaires, dont le maire J. J. E. Guerin et le commissaire Emmanuel Persillier Lachapelle. De leur côté, les pharmaciens sont, d'une certaine façon, des commerçants.

Quant aux gestionnaires, aux employés et aux ouvriers (un charretier et un charpentier-menuisier), leur présence au Conseil n'est que symbolique. Parmi ces derniers, le plus connu est le syndicaliste Joseph Ainey, commissaire à partir de 1910. Par contre, les conseillers élus en $1910 \mathrm{ou}$ 1912 avec l'appui du Parti ouvrier ${ }^{23}$ comprennent deux entrepreneurs en construction (Ludger Clément et Noé Leclaire), un entrepreneur plombier (Napoléon Turcot), un avocat (Ernest Tétrau) et un notaire (Georges Mayrand), mais aucun ouvrier salarié.

Comment la répartition professionnelle se manifeste-t-elle de chaque côté de la frontière linguistique? Le tableau 5 indique quelques différences. Comme on peut s'y attendre, les hommes d'affaires dominent nettement chez les anglophones, avec $77 \%$ des professions déclarées, mais leur présence est aussi très forte chez les francophones (70\%). Les commerçants ont un poids comparable dans les deux groupes. Les différences viennent des industriels, proportionnellement plus nombreux chez les anglophones, et des entrepreneurs en construction, dont plus des trois quarts sont francophones. On s'attend aussi à ce que les membres des professions libérales soient plus nombreux parmi les francophones: c'est bien le cas puisqu'ils y représentent $28,5 \%$ des effectifs, contre $15,7 \%$ chez les anglophones.

Il y a également des différences selon les groupes ethniques. Les effectifs irlandais sont pour moitié des commerçants et pour un cinquième des industriels. Parmi les Écossais, les industriels comptent pour un tiers, les commerçants et les entrepreneurs en construction, pour un cinquième chacun. Chez les Anglais, les professions libérales dominent, avec plus du tiers, suivies de près par les commerçants, puis viennent les industriels avec un cinquième ${ }^{24}$. Les commerçants occupent le premier

23. Chronologie des mouvements politiques ouvriers..., 101, 122.

24. Il ne faut pas oublier que 4 des 31 individus d'origine anglaise sont considérés comme des francophones: deux commerçants, un médecin et un avocat. 
rang (35\%) chez les Canadiens français, suivis des membres des professions libérales (28\%), puis des entrepreneurs en construction (18\%).

Cette analyse de l'appartenance professionnelle permet déjà de souligner une caractéristique de la scène politique montréalaise qui la distingue des scènes politiques fédérale et provinciale: le poids beaucoup plus considérable des hommes d'affaires parmi les conseillers municipaux, par rapport aux professions libérales, qui viennent au premier rang chez les députés ${ }^{25}$. Deux hypothèses peuvent être avancées pour expliquer cette situation. La nature des interventions municipales, notamment les lourds investissements dans les infrastructures, a un impact économique qui peut inciter les premiers bénéficiaires à s'y intéresser de près. En outre, la taille plus petite des territoires de représentation, les quartiers, favorise peut-être la participation politique de ceux qui ont des intérêts directs dans l'économie locale.

Dans son article de 1965, Guy Bourassa note la baisse des détenteurs de «hauts postes dans l'administration» au profit de «leurs subalternes», «les administrateurs d'un niveau intermédiaire ${ }^{26} »$. L'auteur n'explique pas la composition de ces catégories ni à partir de quelles informations il les a constituées. Or, les données présentes dans les recueils biographiques ne permettent pas d'afficher une telle certitude. Il est indéniable qu'il existe une hiérarchie parmi les hommes d'affaires. Certains, tel J. J. C. Abbott, sont associés à de grandes sociétés d'envergure canadienne ou internationale, d'autres, comme Hormisdas Laporte ou Alphonse Desjardins, participent à des entreprises de portée régionale ou provinciale, tandis qu'un grand nombre n'œuvrent qu'à l'échelle locale, dans de petits commerces de quartier. Pour établir la position de chacun des hommes d'affaires au sein de cette hiérarchie, il faudra dépasser les mentions professionnelles et examiner en détail l'ensemble des entreprises auxquelles ils sont associés.

Si l'information sur la profession fournit un premier portrait social des élus municipaux, elle reste bien insuffisante pour dresser un tableau complet. Il faudrait aussi évaluer la fortune ou la richesse des individus, tenir compte de leur présence dans les institutions sociales, culturelles, religieuses ou autres et des postes de prestige ou d'influence qu'ils y occupent, reconstituer leurs réseaux familiaux et sociaux. Les répertoires biographiques livrent des informations utiles à cet égard, mais elles sont notoirement incomplètes ou imprécises. Il faudra élargir l'enquête pour

25. Robert Boily, «Les hommes politiques du Québec, 1867-1967», Revue d'histoire de l'Amérique française, 21,3a (1967): 611-614.

26. Guy Bourassa, op. cit., 130. 
parvenir à bien cerner la composition sociale du personnel politique et son évolution pendant la période.

Dans une recherche complémentaire à celle-ci, Christine Simard a examiné les caractéristiques résidentielles des membres du Conseil municipal en 1881 et en $1912^{27}$. On y relève des phénomènes fort intéressants. En 1881, presque tous les élus anglophones habitent le Golden Square Mile et ses environs, dans l'axe de la rue Sherbrooke Ouest, et presque tous les élus francophones se trouvent dans le secteur bourgeois canadien-français, autour de la rue Saint-Denis. C'est donc dire que plusieurs conseillers ne résident pas dans le quartier qu'ils représentent. Le caractère élitiste de la délégation au Conseil en ressort encore plus nettement.

En 1912, le personnel politique se divise en deux blocs égaux, la moitié habitant à l'intérieur des limites anciennes de la ville (celles qui existaient en 1881), l'autre moitié se trouvant dans les nouveaux quartiers, annexés graduellement depuis 1883. Le premier groupe reproduit les caractéristiques qui prévalaient en 1881 et la plupart de ses membres réside dans les deux zones bourgeoises (quelques francophones se retrouvant toutefois dans celle de l'ouest); seule une minorité habite le quartier qu'elle représente. Par constraste, les membres du second groupe sont dispersés dans la plupart des nouveaux quartiers et la quasi-totalité d'entre eux habite dans le quartier qu'elle représente. Qui plus est, les quatre cinquièmes des membres du premier groupe ont une résidence dont la valeur locative annuelle est supérieure à $300 \$$, tandis que, exactement dans la même proportion, les membres du second groupe affichent une valeur locative de $300 \$$ ou moins ${ }^{28}$.

Cela laisse croire que la composition sociale du personnel politique montréalais s'est diversifiée entre ces deux dates. On aurait, d'une part, une élite à l'aise, identifiée au centre-ville, et, d'autre part, une nouvelle élite, plus modeste et enracinée dans les quartiers périphériques, où elle serait plus près de ses électeurs. Ces informations semblent aussi indiquer que les annexions des municipalités de banlieue représentent un facteur important de la transformation politique de Montréal à cette époque.

\section{LA CARRIÈRE POLITIQUE}

L'appartenance professionnelle des élus municipaux laisse deviner que plusieurs ont un intérêt direct dans le développement de la ville ou à

27. Christine Simard, Le personnel politique municipal de Montréal, 1881-1912: espace urbain, richesse et propriété foncière, mémoire de maîtrise (histoire), Université du Québec à Montréal, 1997, $142 \mathrm{p}$.

28. Ibid., 60-92. 
tout le moins de leur quartier. Comment se manifeste leur engagement sur la scène politique montréalaise? S'agit-il d'un phénomène ponctuel ou de longue durée? Le mandat municipal n'est-il qu'un tremplin vers une activité politique à un autre niveau?

Il faut rappeler que l'électorat varie selon le poste occupé. Les maires et les commissaires sont élus par l'ensemble des propriétaires et des locataires montréalais qui ont qualité d'électeur. Les conseilleurs ne doivent leur mandat qu'aux électeurs, propriétaires et locataires, de leur quartier.

Examinons d'abord le cas des 224 conseillers municipaux. La majorité n'a obtenu qu'un seul (26\%) ou que deux (31\%) mandats des électeurs. Mais comme la durée des mandats change en cours de route (avant 1894, elle est de trois ans, à partir de cette date, de deux ans), il vaut mieux recourir au nombre d'années de service au Conseil (tableau 6). Il s'agit ici du nombre total pour chaque conseiller présent entre 1880 et 1914; il prend donc en compte les années de service que certains ont assurées avant ou après cette période. L'éventail des durées est assez considérable et indique une vaste gamme de situations. Un peu plus de la moitié $(53,1 \%)$ des conseillers le furent moins de six ans. Ce phénomène touche plus les francophones $(56,8 \%)$ que les anglophones $(46,1 \%)$ et plus ceux qui sont élus pour la première fois en 1898 ou après $(62,3 \%)$ que ceux qui le sont avant $(44,9 \%)$. Il y a donc manifestement un plus fort roulement au début du $\mathrm{XX}^{\mathrm{e}}$ siècle, ce qui affecte davantage les francophones, alors plus nombreux. Cela peut s'expliquer par les aléas du mouvement réformiste et par les réductions du nombre de conseillers survenues en 1894 et en 1910, mais il faudrait examiner la situation cas par cas pour en être certain. Notons par ailleurs que les situations de longévité politique élevée (10 ans et plus) se répartissent assez également entre les deux groupes linguistiques, bien que les francophones dominent nettement dans le groupe des 16 ans et plus.

Pendant la période, Montréal annexe plusieurs municipalités de banlieue. Or très souvent, les premiers conseillers de ces territoires annexés élus au Conseil municipal sont d'ex-maires ou échevins de leur localité. Les exemples les plus notoires sont ceux des anciens maires d'Hochelaga et de Saint-Jean-Baptiste, Raymond Préfontaine et Joseph Octave Villeneuve, qui deviennent tous deux conseillers, puis maires, à Montréal. La prise en compte de cette variable permettrait de constater que, pour plusieurs, la carrière au niveau municipal est plus longue que celle qui apparaît ici, mais faute d'informations suffisamment complètes, elle n'est pas intégrée à l'analyse. 
TABLEAU 6

\section{NOMBRE TOTAL D'ANNÉES COMME CONSEILLER CONSEILLERS MUNICIPAUX DE MONTRÉAL ENTRE 1880 ET 1914*}

$\begin{array}{lrrrr}\text { Nombre } & \text { Anglophones } & \text { Francophones } & \text { Total } & \% \text { du total } \\ 1 & 3 & 10 & 13 & 5,8 \\ 2 & 11 & 41 & 41 & 18,3 \\ 3 & 9 & 18 & 18 & 8,0 \\ 4 & 11 & 38 & 38 & 17,0 \\ 5 & 2 & 9 & 9 & 4,0 \\ 6 & 11 & 31 & 31 & 13,8 \\ 7 & 2 & 10 & 10 & 4,5 \\ 8 & 6 & 16 & 16 & 7,1 \\ 9 & 2 & 4 & 4 & 1,8 \\ 10 & 2 & 5 & 5 & 2,2 \\ 11-15 & 15 & 24 & 24 & 10,6 \\ 16-20 & 2 & 11 & 11 & 4,8 \\ 21-32 & 2 & 4 & 4 & 1,2 \\ \text { TOTAL } & 78 & 146 & 224 & 100,0\end{array}$

* Ce tableau n'inclut pas les cinq maires et les cinq commissaires qui n'ont jamais été conseillers.

Nous avons calculé l'âge des conseillers au début de leur premier mandat à la Ville de Montréal (tableau 7). Le Conseil municipal n'est manifestement pas un rassemblement de jeunes et l'élection avant 35 ans est exceptionnelle. Faut-il y voir un effet de l'exigence de la qualification foncière? Ce n'est pas non plus le lieu où se manifestent les vocations tardives, car peu d'hommes y font leur entrée après 55 ans. Les trois quarts des nouveaux venus s'y présentent entre 35 et 54 ans et ils sont assez bien répartis entre les tranches d'âges. L'âge moyen à l'entrée est de 44,8 ans. Notons toutefois une différence entre les deux groupes linguistiques: les anglophones sont proportionnellement plus représentés dans les tranches de 45 ans et plus (59\% contre 47,7\% pour l'ensemble du groupe), les francophones dans celles de 44 ans et moins $(56,1 \%$, contre $50 \%)$.

La carrière des maires se présente différemment de celle des conseillers. Avant 1894, les élections à la mairie ont lieu tous les ans et la plupart obtiennent deux mandats, à l'exception d'Alphonse Desjardins qui n'en sollicite qu'un et de Jean-Louis Beaudry qui monopolise le poste 
TABLEAU 7

\section{ÂGE AU DÉBUT DU PREMIER MANDAT PAR GROUPE D'ÂGES CONSEILLERS MUNICIPAUX DE MONTRÉAL ENTRE 1880 ET 1914*}

$\begin{array}{lrcrc}\text { Groupes d'âges } & \text { Anglophones } & \text { Francophones } & \text { Total } & \% \text { du total } \\ <30 & 1 & 1 & 2 & 0,9 \\ 30 \text { à 34 } & 5 & 19 & 24 & 10,7 \\ 35 \text { à 39 } & 12 & 31 & 43 & 19,2 \\ 40 \text { à } 44 & 12 & 31 & 43 & 19,2 \\ 45 \text { à } 49 & 16 & 28 & 44 & 19,6 \\ 50 \text { à } 54 & 13 & 21 & 34 & 15,2 \\ 55 \text { à } 59 & 11 & 9 & 20 & 8,9 \\ 60 \text { et plus } & 6 & 3 & 9 & 4,0 \\ \text { Indéterminée } & 2 & 3 & 5 & 2,2 \\ \text { TOTAL } & 78 & 146 & 224 & 100,0\end{array}$

* Ce tableau n'inclut pas les cinq maires et les cinq commissaires qui n'ont jamais été conseillers.

pendant 10 ans (dont 6 avant 1880). À partir de 1894, les mandats sont de deux ans et tous n'en remplissent qu'un, sauf Raymond Préfontaine qui en a deux. Ainsi, la plupart des 16 individus qui occupent la mairie de 1880 à 1914 n'y sont que pour deux ans. Lors de leur accession à ce poste, ils ont en moyenne 52,5 ans: le plus jeune a 37 ans, trois sont dans la quarantaine, dix dans la cinquantaine et le plus âgé a 66 ans. Il faut ajouter que 11 des maires ont précédemment été conseillers municipaux pour des périodes plus ou moins longues.

Les commissions échevinales ou comités, comme on les désigne alors, représentent des lieux de pouvoir importants au sein du Conseil. Chaque comité gère l'un des services municipaux, ou un ensemble de services, et le budget afférent. Ce régime dure jusqu'en 1910, alors que le Bureau des Commissaires prend la relève. La présidence des comités est particulièrement recherchée. C'est par exemple grâce à son poste de président du comité des chemins, celui qui gère le plus gros budget, que Raymond Préfontaine peut mettre en place la «machine» politique qui le rendra célèbre. Au total, 89 conseillers (dont 5 seront aussi maires), soit $40 \%$ des effectifs, occupent la présidence de l'un ou l'autre des comités au cours de leur carrière. Parmi eux, 38 exercent ces responsabilités pendant quatre ans ou plus et sont presque tous conseillers pendant six ans ou 
plus. Ce petit groupe, $17 \%$ de tous les conseillers, allie pouvoir et longévité et représente à n'en pas douter l'élite du Conseil. Il mérite une analyse plus approfondie qui exigera que l'on tienne compte du poids relatif de chaque comité.

Quant aux cinq commissaires qui sont élus entre 1910 et 1914, leur nombre est trop faible pour qu'on puisse en faire une analyse statistique. Deux d'entre eux ne sont en poste que pour deux ans, deux autres pour quatre ans et le dernier y restera huit ans. Aucun n'a auparavant été conseiller.

Le Conseil municipal de Montréal constitue par ailleurs une véritable pépinière pour les partis qui œuvrent aux échelons supérieurs. Au niveau fédéral, on trouve 15 députés, dont 4 sont en outre députés à Québec et un deviendra conseiller législatif. Quatre d'entre eux seront ministres, dont un (J. J. C. Abbott) premier ministre du Canada. Il y a aussi deux sénateurs qui ont été députés au préalable, l'un à Ottawa, l'autre à Québec. Par ailleurs, 12 autres membres du Conseil municipal sont candidats aux élections fédérales, mais connaissent la défaite, bien que deux d'entre eux réussissent à se faire élire à Québec.

Au niveau provincial, la moisson est encore plus forte puisque 25 députés proviennent du Conseil municipal, dont 4 sont aussi députés à Ottawa et un deviendra sénateur. Neuf d'entre eux sont faits ministres, dont un premier ministre du Québec (Lomer Gouin). On compte aussi 8 conseillers législatifs dont 3 ont déjà été députés. Ajoutons-y 5 candidats défaits.

Au total, 41 individus ont donc occupé des postes de député, de sénateur ou de conseiller législatif: 24 libéraux et 17 conservateurs. Si on y ajoute les 15 candidats malchanceux, on obtient 56 personnes, soit près du quart de l'effectif total du personnel politique municipal de la période. Il est probable que de nombreux autres ont été à l'œuvre au sein des grands partis à titre d'organisateurs ou de conseillers. L'affiliation politique d'un peu plus de la moitié des membres du Conseil est connue: 62 sont conservateurs, 59 sont libéraux et 4 se déclarent indépendants. Parmi les cinq conseillers élus avec l'appui du Parti ouvrier, trois sont par ailleurs identifiés comme libéraux.

Il n'y a donc pas un modèle unique ou dominant de carrière politique à Montréal. Pour un noyau important, il s'agit d'une véritable profession qui se pratique à deux ou même trois niveaux de gouvernement. En outre, le mandat municipal est souvent exercé concurremment à celui du niveau supérieur. Le politicien par excellence est sans aucun doute Raymond Préfontaine qui, en plus d'avoir été conseiller et maire d'Hochelaga, est 
conseiller municipal à Montréal pendant 14 ans, dont 9 comme président d'un comité, maire pendant 4 ans, député provincial pendant 5 ans, député fédéral pendant 19 ans, dont 3 comme ministre. Parmi les députés et les ministres, plusieurs ont à leur actif une longue prestation comme conseiller: Joseph Brunet, 21 ans, Henri-Benjamin Rainville, 18, James McShane, 17, Hormisdas Jeannotte et Louis Audet Lapointe, 16, Cléophas Beausoleil, Patrick Kennedy et George Washington Stephens, 14 ans. Par contre, certains élus d'un niveau supérieur ne font qu'une brève apparition sur la scène municipale: c'est le cas pour J. J. C. Abbott, Alphonse Desjardins et Lomer Gouin.

Il reste néanmoins que, pour plus des trois quarts des élus, la carrière publique est entièrement consacrée au niveau municipal. On observe parmi eux de nombreux exemples de longévité politique. Jacques Grenier siège 26 ans comme conseiller (en plus d'être maire), A. A. Stevenson, 24 ans, Émery-François Larivière, 18, Peter Donovan et Arthur Dubuc, 14, Joseph B. Clearihue, 12, Louis-Arsène Lavallée, 11 (et 2 ans comme maire), Napoléon Giroux et Jean-Damien Rolland, 10 ans; tous jouissent d'une influence certaine et sont aussi présidents d'un comité pendant une partie de leur mandat. Il y en a de nombreux autres dans ce cas. Certains n'obtiennent aucune présidence, mais servent pendant longtemps leurs électeurs comme simples conseillers: Richard Turner, 16 ans, George William Sadler, 14 ans, John Bumbray, 11 ans ou Philéas Wilson, 8 ans, en sont quelques exemples.

Le Conseil municipal est cependant aussi témoin de nombreuses carrières éphémères, souvent de deux ou de quatre ans. Le départ de ces conseillers est-il causé par une défaite électorale, par un décès, par la perte de la qualification foncière ou s'explique-t-il par une autre raison? Il faudrait une analyse cas par cas pour pouvoir y répondre.

L'étude de la scène municipale est certainement la parente pauvre de l'histoire politique au Québec. Or notre recherche montre qu'il existe une véritable carrière politique municipale, souvent couronnée par une présidence d'un comité du Conseil. Elle n'est peut-être pas le cas de la majorité, bien que l'examen détaillé des postes occupés antérieurement dans les municipalités de banlieue permettrait peut-être d'évaluer différemment les durées. Elle concerne tout de même un nombre suffisamment important d'élus pour qu'on lui accorde plus d'attention qu'on ne l'a fait jusqu'ici. Cette constation amène d'ailleurs à s'interroger sur la nature de la «professionnalisation» de la carrière politique qui, selon les politologues, apparaît après la Première Guerre mondiale. À Montréal, elle semble s'être manifestée bien avant. La longévité politique, en tout cas, n'est 
pas un apanage du $\mathrm{XX}^{\mathrm{e}}$ siècle et de nombreux conseillers paraissent très ancrés dans leur milieu. Par ailleurs, l'analyse confirme, tout en la précisant, celle de Guy Bourassa au sujet de la présence significative des élus municipaux aux niveaux provincial et fédéral.

\section{CONCLUSION}

Ces premiers résultats de l'enquête sur le personnel politique municipal à Montréal entre 1880 et 1914 restent évidemment partiels. Il sont tout de même suffisamment intéressants pour que l'on tente d'en dégager quelques traits significatifs.

L'historien Marc V. Levine a étudié La reconquête de Montréal par les francophones à partir de $1960^{29}$. Il faut toutefois constater que cette reconquête s'est étalée sur plus d'un siècle et qu'elle s'est d'abord manifestée dans l'arène politique. L'étude de la période 1880-1914 confirme et précise l'ampleur et le rythme de cette première phase. La domination numérique des Canadiens français au conseil municipal devient de plus en plus marquée à mesure qu'on avance vers le $\mathrm{XX}^{\mathrm{e}}$ siècle. Cette évolution se produit en parallèle avec l'émergence de nouvelles élites francophones au sein de la société civile. Particulièrement notable est l'affirmation d'une bourgeoisie d'affaires dont la présence se fait aussi sentir au Conseil municipal. Les hommes d'affaires forment en effet $70 \%$ du personnel politique francophone et ce pourcentage n'est pas tellement inférieur à celui qui prévaut chez les anglophones. L'association étroite que l'on fait souvent entre politicien canadien-français et membre d'une profession libérale ne convient pas ici. Certes, les effectifs des professions libérales vont croissant et sont plus élevés que chez les anglophones, mais pas assez pour que cela devienne la caractéristique spécifique des élus francophones.

Corollaire du phénomène précédent, la part des anglophones décline au cours de la période. Une cassure importante, vers la fin des années 1880, a été identifiée. Des différences significatives selon les groupes ethniques ont aussi été mises en lumière. Les Irlandais, dont on ne soupçonnait pas le poids politique pour les années 1880 , sont les plus nettement touchés par ce déclin, tandis que les Anglais et les Écossais maintiennent mieux leurs positions et voient leur part baisser de façon moins abrupte.

Il ressort aussi que les membres du personnel politique sont majoritairement et de plus en plus originaires du Québec et que le poids des immigrants décline nettement à mesure que l'on s'approche du $\mathrm{XX}^{\mathrm{e}}$ siè-

29. Marc V. Levine, La reconquête de Montréal (Montréal, VLB éditeur, 1997), 404 p. 
cle. Les Montréalais de naissance ne sont qu'une minorité à une époque où l'exode rural forme une composante importante de la croissance urbaine. Le territoire de ces migrations est cependant bien délimité: c'est la grande région de Montréal qui fournit à la Ville le plus gros de son personnel politique.

L'étude a également permis de constater que le lien entre la politique et les affaires reste très étroit avant 1914. La nette prépondérance des hommes d'affaires le confirme sans l'ombre d'un doute. Il faudra cependant établir la nature et l'ampleur de leurs activités pour arriver à mieux cerner la hiérarchie au sein de ce groupe et pour voir comment elle évolue. Le poids des professions libérales augmente, mais sans devenir prédominant, et plusieurs de leurs représentants sont des participants actifs au monde des affaires. Par contre, les couches sociales en émergence les ingénieurs, les architectes, les comptables et les cadres salariés sont à peu près absentes du Conseil municipal, tout comme les ouvriers et les employés. Ainsi, le Conseil reflète bien mal la composition sociale de la population montréalaise.

Le personnel politique montréalais constitue clairement une élite qui s'alimente à certaines des élites sectorielles de la société civile. Le Conseil municipal représente un lieu de rencontre de membres des élites montréalaises, celles des principaux groupes ethniques et linguistiques et celles des groupes professionnels dominants. Il est encore un lieu de pouvoir recherché où s'opposent et s'allient des intérêts divers et parfois divergents.

Cette élite politique a toutefois une spécificité propre, dans la mesure où elle attire de nombreux individus pour qui la vie publique forme la base d'une seconde carrière, distincte de la profession principale tout en ayant des liens évidents avec celle-ci. Elle donne même naissance à une élite plus restreinte qui accède aux postes de pouvoir ou de prestige, tels ceux de président d'un comité ou de maire. L'étude de ce noyau devrait permettre de mieux connaître les caractéristiques des détenteurs du pouvoir politique.

Les données biographiques, même partielles, recueillies au cours de l'enquête ne se limitent pas aux phénomènes présentés ici. En examinant les mariages et les autres liens de parenté, la participation à la vie associative, la présence aux conseils d'administration, en scrutant de façon plus fine le déroulement des carrières individuelles ou encore l'accumulation de propriétés, il sera possible de mettre en lumière d'autres phénomènes plus complexes et de faire ressortir les réseaux de parenté, 
d'affaires, de sociabilité ou de pouvoir qui se constituent dans la société montréalaise de l'époque.

Cependant, même si on parvient à lui faire livrer tous ses fruits, une enquête de type prosopographique présente de sérieuses limites et ne peut répondre à toutes les questions. Ainsi, pour comprendre la présence au Conseil de tel ou tel individu, on ne peut se reposer uniquement sur ses données biographiques. Il faut connaître le milieu dans lequel il agit et qu'il représente. Il faut savoir qui sont ses électeurs et quel appui ils lui donnent, examiner ses positions dans les débats, les politiques qu'il défend ou qu'il combat. La prosopographie n'éclaire qu'une partie d'un processus politique plus vaste, dont la reconstitution historique requiert la mise en œuvre de sources et de méthodes variées.

La mise en lumière, par la prosopographie, de phénomènes collectifs d'appartenance ne doit pas amener à en déduire des comportements collectifs. Constater la montée des francophones, par exemple, est une chose; en examiner la portée et les conséquences en est une autre. Francophones et anglophones forment-ils des blocs compacts et étanches? En certaines circonstances, oui, mais le plus souvent, non. C'est qu'interviennent aussi toutes les autres appartenances: religieuses, sociales, professionnelles, géographiques, politiques, etc. Michèle Dagenais en offre un bon exemple dans son récit des discussions interminables - tant sur le principe que sur les modalités ou le site — qui ont mené à la décision de construire la bibliothèque municipale ${ }^{30}$. Il faudrait multiplier les analyses de ces débats politiques locaux pour arriver à mieux comprendre le poids des diverses appartenances et le jeu complexe des alliances.

Qui plus est, si le Conseil municipal offre une excellente vitrine pour observer les tensions et les alliances, il ne saurait épuiser la réalité du phénomène politique au niveau local. Les rapports de pouvoir s'expriment en bien d'autres lieux et les élites ont d'autres moyens pour faire valoir leurs points de vue. Ces élites sont tout autant divisées que le Conseil luimême. David C. Hammack l'a bien montré dans son ouvrage sur New York à la même époque en constatant que «Altogether, Greater New York's economic and social elites were divided so deeply and in so many ways that they could not agree on any single set of economic, cultural, or

30. Michèle Dagenais, «Vie culturelle et pouvoirs politiques locaux. La fondation de la bibliothèque municipale de Montréal», Urban History Review/Revue d'histoire urbaine, 24,2 (mars 1996): 41-56. 
political initatives ${ }^{31}$.» Ce que nous savons de Montréal nous incite à penser que la situation n'y est pas tellement différente.

Les premiers résultats de l'étude de la biographie collective des élus municipaux permettent toutefois de lever un coin du voile entourant le portrait complexe du pouvoir et des élites à Montréal et font la preuve de l'utilité de la méthode prosopographique.

\section{ANNEXE 1}

\section{Principaux répertoires biographiques utilisés}

ATHERTON, W. H., Montreal 1535-1914. Volume III. Biographical, 1914.

Biographies canadiennes-françaises. Diverses années, 1920 à 1931.

BORTHWICK, J. D., History and Biographical Gazetteer of Montreal, 1892.

Canadian Parliamentary Companion. Diverses années, 1863 à 1885.

COCHRANE, W. The Canadian Album. Men of Canada. 1893-1896. Vol. II à V.

Dictionnaire biographique du Canada. 1982-1994. Vol. XI à XIII.

Guide parlementaire canadien. Diverses années, 1901-1938.

Industries of Canada. City of Montreal. 1886.

JOHNSON, J. Keith., Canadian Directory of Parliament, 1867-1967.

LAMOTHE, J. Cléophas, Histoire de la Corporation de la Cité de Montréal depuis son origine jusqu'à nos jours, 1903.

MONTREAL BOARD OF TRADE, A Souvenir of the Opening of the New Building, 1893.

Montréal fin-de-siècle, 1899.

Montreal Illustrated, 1894.

Montreal of To-Day, 1896.

MORGAN, Henry James, The Canadian Men and Women of the Time. Éditions de 1898 et 1912.

PRINCE, Lorenzo, Montreal Old and New, 1915.

31. David C. Hammack, Power and Society: Greater New York at the Turn of the Century (New York, Russell Sage Foundation, 1982), 306. 
Prominent People of the Province of Quebec, 1923-1924.

Répertoire des parlementaires québécois 1867-1978, 1980.

ROSE, G. M., A Cyclopedia of Canadian Biography, 1886-1888.

SAINT-PIERRE, T., Histoire du commerce canadien-français de Montréal 1535-1893, 1894.

Souvenir de Maisonneuve, 1896.

TERRILL, F. W., A Chronology of Montreal, 1893.

WOOD, William, The Storied Province of Quebec, 1931-1932. Vol. 3 à 5. 